\title{
Adaptive Exponential Stabilization for a Class of Stochastic Nonholonomic Systems
}

\author{
Xiaoyan Qin \\ College of Mathematics and Statistics, Zaozhuang University, Zaozhuang 277160, China \\ Correspondence should be addressed to Xiaoyan Qin; qin-xiaoyan@163.com
}

Received 21 July 2013; Revised 22 October 2013; Accepted 22 October 2013

Academic Editor: Mark McKibben

Copyright (C) 2013 Xiaoyan Qin. This is an open access article distributed under the Creative Commons Attribution License, which permits unrestricted use, distribution, and reproduction in any medium, provided the original work is properly cited.

\begin{abstract}
This paper investigates the adaptive stabilization problem for a class of stochastic nonholonomic systems with strong drifts. By using input-state-scaling technique, backstepping recursive approach, and a parameter separation technique, we design an adaptive state feedback controller. Based on the switching strategy to eliminate the phenomenon of uncontrollability, the proposed controller can guarantee that the states of closed-loop system are global bounded in probability.
\end{abstract}

\section{Introduction}

The nonholonomic systems cannot be stabilized by stationary continuous state feedback, although it is controllable, due to Brockett's theorem [1]. So the well-developed smooth nonlinear control theory and the method cannot be directly used in these systems. Many researchers have studied the control and stabilization of nonholonomic systems in the nonlinear control field and obtained some success [26]. It should be mentioned that many literatures consider the asymptotic stabilization of nonholonomic systems; the exponential convergence is also an important topic theme, which is demanded in many practical applications. However, the exponential regulation problem, particularly the systems with parameterization, has received less attention. Recently, [3] firstly introduced a class of nonholonomic systems with strong nonlinear uncertainties and obtained global exponential regulation. References $[4,5]$ studied a class of nonholonomic systems with output feedback control. Reference [6] combined the idea of combined input-statescaling and backstepping technology, achieving the asymptotic stabilization for nonholonomic systems with nonlinear parameterization.

It is well known that when the backstepping designs were firstly introduced, the stochastic nonlinear control had obtained a breakthrough [7]. Based on quartic Lyapunov functions, the asymptotical stabilization control in the large of the open-loop system was discussed in [8]. Further research was developed by the recent work [9-16]. [17-19] studied a class of nonholonomic systems with stochastic unknown covariance disturbance. Since stochastic signals are very prevalent in practical engineering, the study of nonholonomic systems with stochastic disturbances is very significant. So, there exists a natural problem that is how to design an adaptive exponential stabilization for a class of nonholonomic systems with stochastic drift and diffusion terms. Inspired by these papers, we will study the exponential regulation problem with nonlinear parameterization for a class of stochastic nonholonomic systems. We use the inputstate-scaling, the backstepping technique, and the switching scheme to design a dynamic state-feedback controller with $\Sigma^{T} \sum \neq I$; the closed-loop system is globally exponentially regulated to zero in probability.

This paper is organized as follows. In Section 2, we give the mathematical preliminaries. In Section 3, we construct the new controller and offer the main result. In the last section, we present the conclusions.

\section{Problem Statement and Preliminaries}

In this paper, we consider a class of stochastic nonholonomic systems as follows: 


$$
\begin{gathered}
d x_{0}=d_{0}(t) u_{0} d t+f_{0}\left(t, x_{0}\right) d t \\
d x_{i}=d_{i}(t) x_{i+1} u_{0} d t+f_{i}\left(t, x_{0}, \bar{x}_{i}\right) d t+\varphi_{i}\left(\bar{x}_{i}\right) \sum(t) d \omega, \\
i=1, \ldots, n-1, \\
d x_{n}=d_{n}(t) u_{1} d t+f_{n}\left(t, x_{0}, x\right) d t+\varphi_{n}(\bar{x}) \sum(t) d \omega,
\end{gathered}
$$

where $x_{0} \in R$ and $x=\left[x_{1}, \ldots, x_{n}\right]^{T} \in R^{n}$ are the system states and $u_{0} \in R$ and $u_{1} \in R$ are the control inputs, respectively. $\bar{x}_{i}=\left[x_{1}, x_{2}, \ldots, x_{i}\right]^{T} \in R^{i},(i=1,2, \ldots, n)$, and $\bar{x}_{n}=x$; $\omega \in R^{r}$ is an $r$-dimensional standard Wiener process defined on the complete probability space $(\Omega, F, P)$ with $\Omega$ being a sample space, $F$ being a filtration, and $P$ being measure. The drift and diffusion terms $f_{i}(\cdot), \varphi_{i}(\cdot)$ are assumed to be smooth, vanishing at the origin $\left(x_{1}, x_{2}, \ldots, x_{i}\right)=(0,0, \ldots, 0)$; $\sum(t): R_{+} \rightarrow R^{r \times r}$ is the Borel bounded measurable functions and is nonnegative definite for each $t \geq 0 . d_{i}(t)$ are disturbed virtual control coefficients, where $i=0,1 \ldots n$.

Next we introduce several technical lemmas which will play an important role in our later control design.

Consider the following stochastic nonlinear system:

$$
d x=f(x, t) d t+g(x, t) d \omega, \quad x(0)=x_{0} \in R^{n},
$$

where $x \in R^{n}$ is the state of system (2), the Borel measurable functions: $f: R^{n+1} \rightarrow R^{n}$ and $g: R^{n+1} \rightarrow R^{n \times r}$ are assumed to be $C^{1}$ in their arguments, and $\omega \in R^{r}$ is an $r$-dimensional standard Wiener process defined on the complete probablity space $(\Omega, F, P)$.

Definition 1 (see [8]). Given any $V(x, t) \in C^{1,2}$, for stochastic nonlinear system (2), the differential operator $L$ is defined as follows:

$$
L V(x, t)=\frac{\partial V}{\partial t}+\frac{\partial V}{\partial x} f+\frac{1}{2} \operatorname{tr}\left(g^{T} \frac{\partial^{2} V}{\partial x^{2}} g\right),
$$

where $C^{1,2}\left(R^{n} \times R_{+} ; R_{+}\right)$denotes all nonnegative functions $V(x, t)$ on $R^{n} \times R_{+}$, which are $C^{1}$ in $t$ and $C^{2}$ in $x$, and for simplicity, the smooth function $f(\cdot)$ is denoted by $f$.

Lemma 2 (see [8]). Let $x$ and $y$ be real variables. Then, for any positive integers $m, n$, and any real number $\varepsilon>0$, the following inequality holds:

$$
\begin{gathered}
\alpha(\cdot) x^{m} y^{n} \leq \varepsilon|x|^{m+n}+\frac{n}{m+n}\left(\frac{m+n}{m}\right)^{-m / n} \\
\times \alpha(\cdot)^{(m+n) / n} \varepsilon^{-m / n}|y|^{m+n} .
\end{gathered}
$$

Lemma 3 (see [7]). Considering the stochastic nonlinear system (2), if there exist a $C^{1,2}$ function $V(x, t), K_{\infty}$ class functions $\underline{\alpha}$ and $\bar{\alpha}$, constant $\bar{c}$, and a nonnegative functions $W(x, t)$ such that

$$
\underline{\alpha}|(x)| \leq V(x) \leq \bar{\alpha}|(x)|, \quad L V(x) \leq-W(x, t)+\bar{c},
$$

then for each $x_{0} \in R^{n}$. (1) For (2), there exists an almost surely unique solution on $[0, \infty]$. (2) When $\bar{c}=0, f(0, t)=0$, $g(0, t)=0$, and $W(x, t)=W(x)$ is continuous, the equilibrium $x=0$ is globally stable in probability, and the solution $x(t)$ satisfies $P\left\{\lim _{t \rightarrow \infty} W(x(t)=0\}=1\right.$. (3) For any given $\varepsilon>0$, there exist a class KL function $\beta_{c}(\cdot, \cdot)$ and $K$ function $\gamma(\cdot)$ such that $P\left\{|(x(t))|<\beta_{c}\left(\left|x_{0}\right|, t\right)+\gamma(c)\right\} \geq 1-\varepsilon$ for any $t \geq 0$, $x_{0} \in R^{n} \backslash\{0\}$.

Lemma 4 (see [20]). For any real-valued continuous function $f(x, y), x \in R^{m}, y \in R^{n}$, there exist smooth scalar-value funcions $a(x) \geq 0, b(y) \geq 0, c(x)>1$, and $d(y) \geq 1$, such that $|f(x, y)| \leq a(x)+b(y)$, and $|f(x, y)| \leq c(x) d(y)$.

\section{Controller Design and Analysis}

The purpose of this paper is to construct a smooth statefeedback control law such that the solution process of system (1) is bounded in probability. For clarity, the case that $x_{0}\left(t_{0}\right) \neq 0$ is firstly considered. Then, the case where the initial $x_{0}\left(t_{0}\right)=0$ is dealt with later. The triangular structure of system (1) suggests that we should design the control inputs $u_{0}$ and $u_{1}$ in two separate stages.

To design the controller for system (1), the following assumptions are needed.

Assumption 5. For $0 \leq i \leq n$, there are some positive constants $\lambda_{i 1}$ and $\lambda_{i 2}$ that satisfy the inequality $\lambda_{i 1} \leq d_{i}(t) \leq$ $\lambda_{i 2}$.

Assumption 6. For $f_{0}\left(t, x_{0}\right)$, there exists a nonnegative smooth function $\gamma_{0}\left(t, x_{0}\right)$, such that $\left|f_{0}\left(t, x_{0}\right)\right| \leq\left|x_{0}\right| \gamma_{0}\left(t, x_{0}\right)$.

For each $f_{i}\left(t, x_{0}, \bar{x}_{i}\right), \varphi_{i}\left(\bar{x}_{i}\right)$, there exist nonnegative smooth functions $\gamma_{i}\left(t, x_{0}, \bar{x}_{i}\right)$ and $\rho_{i}\left(\bar{x}_{i}\right)$, such that $\left|f_{i}\left(t, x_{0}, \bar{x}_{i}\right)\right| \leq\left(\sum_{k=1}^{i}\left|x_{k}\right|\right) \gamma_{i}\left(t, x_{0}, \bar{x}_{i}\right),\left|\varphi_{i}\left(\bar{x}_{i}\right)\right| \leq\left(\sum_{k=1}^{i}\left|x_{k}\right|\right)$ $\rho_{i}\left(\bar{x}_{i}\right)$.

3.1. Designing $u_{0}$ for $x_{0}$-Subsystem. For $x_{0}$-subsystem, the control $u_{0}$ can be chosen as

$$
u_{0}=-\lambda_{0} x_{0}
$$

where $\lambda_{0}=\left(k_{0}+\gamma_{0}\right) / \lambda_{01}$ and $k_{0}$ is a positive design parameter.

Consider the Lyapunov function candidate $V_{0}=x_{0}^{2} / 2$. From (6) and Assumptions 5 and 6, we have

$$
\begin{aligned}
L V_{0} & =x_{0}\left(d_{0} u_{0}+f_{0}\left(t, x_{0}\right)\right) \\
& \leq d_{0} u_{0} x_{0}+x_{0}^{2} \gamma_{0} \leq-k_{0} x_{0}^{2}=-2 k_{0} V_{0} .
\end{aligned}
$$

So, we obtain the first result of this paper.

Theorem 7. The $x_{0}$-subsystem, under the control law (6) with an appropriate choice of the parameters $k_{0}, \lambda_{01}, \lambda_{02}$, is globally exponentially stable.

Proof. Clearly, from (7), $L V_{0} \leq 0$, which implies that $\left|x_{0}(t)\right| \leq$ $\left|x_{0}\left(t_{0}\right)\right| e^{-k_{0}\left(t-t_{0}\right)}$. Therefore, $x_{0}$ is globally exponentially convergent. Consequently, $x_{0}$ can be zero only at $t=t_{0}$, when 
$x\left(t_{0}\right)=0$ or $t=\infty$. It is concluded that $x_{0}$ does not cross zero for all $t \in\left(t_{0}, \infty\right)$ provided that $x\left(t_{0}\right) \neq 0$.

Remark 8. If $x\left(t_{0}\right) \neq 0, u_{0}$ exists and does not cross zero for all $t \in\left(t_{0}, \infty\right)$ independent of the $x$-subsystem from (6).

3.2. Backstepping Design for $u_{1}$. From the above analysis, the $x_{0}$-state in (1) can be globally exponentially regulated to zero as $t \rightarrow \infty$, obviously. In this subsection, we consider the control law $u_{1}$ for the $x$-subsystem by using backstepping technique. To design a state-feedback controller, one first introduces the following discontinuous input-state-scaling transformation:

$$
\eta_{i}=\frac{e^{\alpha t} x_{i}}{u_{0}^{n-i}}, \quad i=1 \ldots, n, u=e^{\alpha t} u_{1} .
$$

Under the new $x$-coordinates, $x$-subsystems is transformed into

$$
\begin{aligned}
& d \eta_{i}=d_{i} \eta_{i+1} d t+\bar{f}_{i} d t+\phi_{i}^{T} \sum^{T}(t) d \omega, \quad i=1, \ldots, n-1, \\
& d \eta_{n}=d_{n} u d t+\bar{f}_{n} d t+\phi_{n}^{T} \sum^{T}(t) d \omega
\end{aligned}
$$

where

$$
\begin{gathered}
\bar{f}_{i}=\alpha \eta_{i}+\frac{e^{\alpha t} f_{i}}{u_{0}^{n-i}}-\frac{(n-i) \eta_{i}}{u_{0}} \frac{\partial u_{0}}{\partial x_{0}}\left(d_{0} u_{0}+f_{0}\right), \\
\phi_{i}=\frac{e^{\alpha t} \varphi_{i}}{u_{0}^{n-i}} .
\end{gathered}
$$

In order to obtain the estimations for the nonlinear functions $\bar{f}_{i}$ and $\phi_{i}$, the following Lemma can be derived by Assumption 6.

Lemma 9. For $i=1,2 \ldots n$, there exist nonnegative smooth functions $\bar{\gamma}_{i}(\cdot), \bar{\rho}_{i}(\cdot)$, such that

$$
\begin{gathered}
\left|\bar{f}_{i}\right| \leq\left(\sum_{k=1}^{i}\left|\eta_{k}\right|\right) \bar{\gamma}_{i}\left(x_{0}, \bar{x}_{i}\right), \\
\left|\phi_{i}\right| \leq\left(\sum_{k=1}^{i}\left|\eta_{k}\right|\right) \bar{\rho}_{i}\left(\bar{x}_{i}\right) .
\end{gathered}
$$

Proof. We only prove (11). The proof of (12) is similar to that of (11). In view of (6), (8), (10) and Assumption 6, one obtains

$$
\begin{aligned}
\left|\bar{f}_{i}\right|= & \left|\alpha \eta_{i}+\frac{e^{\alpha t} f_{i}}{u_{0}^{n-i}}-\frac{(n-i) \eta_{i}}{u_{0}} \frac{\partial u_{0}}{\partial x_{0}}\left(d_{0} x_{0}+f_{0}\right)\right| \\
\leq & \left|\alpha \eta_{i}\right|+\left(\sum_{k=1}^{i} \frac{e^{\alpha t}\left|x_{k}\right|}{u_{0}^{n-k}}\left|u_{0}^{i-k}\right|\right) \gamma_{i} \\
& +(n-i)\left(\lambda_{0} \lambda_{02}+\gamma_{0}\right)\left|\eta_{i}\right|
\end{aligned}
$$

$$
\begin{aligned}
& \leq|\alpha|\left|\eta_{i}\right|+\left(\sum_{k=1}^{i}\left|\eta_{k}\right| \lambda_{0}^{i-k}\left|x_{0}^{i-k}\right|\right) \gamma_{i} \\
& \quad+(n-i)\left(\lambda_{0} \lambda_{02}+\gamma_{0}\right)\left|\eta_{i}\right| \\
& \leq\left(\sum_{k=1}^{i}\left|\eta_{k}\right|\right)\left(|\alpha|+\left|\lambda_{0}^{i-k}\right|\left|x_{0}^{i-k}\right| \gamma_{i}+(n-i)\left(\lambda_{0} \lambda_{02}+\gamma_{0}\right)\right) \\
& \leq\left(\sum_{k=1}^{i}\left|\eta_{k}\right|\right) \bar{\gamma}_{i}\left(x_{0}, \bar{x}_{i}\right)
\end{aligned}
$$

where $\bar{\gamma}_{i}\left(x_{0}, \bar{x}_{i}\right) \geq|\alpha|+\left|\lambda_{0}^{i-k}\right| x_{0}^{i-k} \mid \gamma_{i}+(n-i)\left(\lambda_{0} \lambda_{02}+\gamma_{0}\right)$.

To design a state-feedback controller, one introduces the coordinate transformation

$$
\begin{gathered}
z_{1}=\eta_{1}, \\
z_{i}=\eta_{i}-\alpha_{i}\left(\bar{z}_{i-1}\right), \quad i=1,2 \ldots, n,
\end{gathered}
$$

where $\alpha_{2}, \ldots, \alpha_{n}$ are smooth virtual control laws and will be designed later and $\alpha_{1}=0 . \widehat{\theta}$ denotes the estimate of $\theta$, where

$$
\begin{gathered}
\theta=\sup _{t \geq 0}\left\{\operatorname { m a x } \left\{\left\|\sum(t) \sum^{T}(t)\right\|^{2},\left\|\sum(t) \sum^{T}(t)\right\|^{4 / 3},\right.\right. \\
\left.\left.\left\|\sum(t) \sum^{T}(t)\right\|\right\}\right\} .
\end{gathered}
$$

Then using (9), (10), (14) and It ô differentiation rule, one has

$$
\begin{aligned}
& d z_{i}= d\left(\eta_{i}-\alpha_{i}\right) \\
&=\left(d_{i} \eta_{i+1}+F_{i}\left(\bar{z}_{i}, x_{0}\right)-\frac{\partial \alpha_{i}}{\partial \hat{\theta}}\right) d t+G_{i}^{T}\left(\bar{z}_{i}\right) \sum^{T}(t) d \omega \\
&-\frac{1}{2} \sum_{k, m=1}^{i-1} \frac{\partial^{2} \alpha_{i}}{\partial z_{k} \partial z_{m}} \phi_{k}^{T}\left(\bar{z}_{k}\right) \sum^{T}(t) \sum(t) \phi_{m}\left(\bar{z}_{m}\right) d t, \\
& i=1,2 \ldots n,
\end{aligned}
$$

where $\eta_{n+1}=u, F_{i}\left(\bar{z}_{i}, x_{0}\right)=\bar{f}_{i}+\sum_{k=1}^{i-1}\left(\partial \alpha_{i} / \partial z_{k}\right)\left(d_{k} \eta_{k+1}+\bar{f}_{k}\right)$, and $G_{i}\left(\bar{z}_{i}, x_{0}\right)=\phi_{i}+\sum_{k=1}^{i-1}\left(\partial \alpha_{i} / \partial z_{k}\right) \phi_{k}$, where $i=1,2 \ldots n$. Using Lemmas 2, 4, and 9 and (14), we easily obtain the following lemma.

Lemma 10. For $1 \leq i \leq n$, there exist nonnegative smooth functions $\gamma_{i 1}\left(\bar{z}_{i}, x_{0}\right), p_{i 1}\left(\bar{z}_{i}\right)$, and $\bar{p}_{i}\left(\bar{z}_{i}\right)$, such that

$$
\begin{aligned}
& \left|F_{i}\right| \leq\left(\sum_{k=1}^{i}\left|z_{k}\right|\right) \gamma_{i 1}\left(\bar{z}_{i}, x_{0}\right), \\
& \left|G_{i}\right| \leq\left(\sum_{k=1}^{i}\left|z_{k}\right|\right) p_{i 1}\left(\bar{z}_{i}\right), \\
& \left|\Phi_{i}\right| \leq\left(\sum_{k=1}^{i}\left|z_{k}\right|\right) \bar{p}_{i}\left(\bar{z}_{i}\right) .
\end{aligned}
$$


The proof of Lemma 10 is similar to that of Lemma 9, so we omitted it.

We now give the design process of the controller.

Step 1. Consider the first Lyapunov function $V_{1}\left(z_{1}, \widehat{\theta}\right)=$ $(1 / 4) z_{1}^{4}+(1 / 2)(\widehat{\theta}-\theta)^{2}$. By (14), (15), and (16), we have

$$
\begin{aligned}
L V_{1}= & z_{1}^{3}\left(d_{1} \eta_{2}+F_{1}\right)+\frac{3}{2} z_{1}^{2} \operatorname{Tr}\left(G_{1}^{T} \sum^{T}(t) \sum(t) G_{1}\right) \\
& +(\widehat{\theta}-\theta) \dot{\hat{\theta}}
\end{aligned}
$$

Using Lemma 10 and Lemma 4, we have

$$
\begin{gathered}
\left|z_{1}^{3} F_{1}\right| \leq z_{1}^{4} \gamma_{11}\left(z_{1}, x_{0}\right) \\
\left|\frac{3}{2} z_{1}^{2} \operatorname{Tr}\left(G_{1}^{T} \sum^{T}(t) \sum(t) G_{1}\right)\right| \\
\leq z_{1}^{4} p_{11}^{2}\left(z_{1}, x_{0}\right)\left|\sum^{T}(t) \sum(t)\right| \leq z_{1}^{4} p_{11}^{2}\left(z_{1}, x_{0}\right) \theta .
\end{gathered}
$$

Substituting (19) into (18) and using (14), we have

$$
\begin{aligned}
L V_{1} \leq & d_{1} z_{1}^{3}\left(\eta_{2}-\alpha_{2}\right)+d_{1} z_{1}^{3} \alpha_{2}+z_{1}^{4} p_{11}^{2}\left(z_{1}, x_{0}\right) \theta \\
& +z_{1}^{4} \gamma_{11}\left(z_{1}, x_{0}\right)+(\widehat{\theta}-\theta) \dot{\hat{\theta}} \\
\leq & d_{1} z_{1}^{3} z_{2}+d_{1} z_{1}^{3} \alpha_{2}+z_{1}^{4} p_{11}^{2}\left(z_{1}, x_{0}\right) \theta \\
& +z_{1}^{4} \gamma_{11}\left(z_{1}, x_{0}\right)+(\hat{\theta}-\theta) \dot{\hat{\theta}}
\end{aligned}
$$

where $\alpha_{2}=-z_{1} \beta_{1}=-z_{1}\left(\left(c_{1}+\gamma_{11}+p_{11}^{2} \widehat{\theta}\right) / \lambda_{11}\right)$. Substituting $\alpha_{2}$ into (20), we have

$$
L V_{1} \leq d_{1} z_{1}^{3} z_{2}-c_{1} z_{1}^{4}+(\widehat{\theta}-\theta)\left(\dot{\hat{\theta}}-\tau_{1}\right)
$$

where $\tau_{1}=z_{1}^{4} p_{11}^{2}$.

Step i. $(2 \leq i \leq n)$. Assume that at step $i-1$, there exists a smooth state-feedback virtual control $\alpha_{i}=$ $-z_{i-1} \beta_{i-1}\left(\bar{z}_{i-1}, \widehat{\theta}\right)=-z_{i-1}\left(\left(c_{i-1}+\widehat{\theta} \sqrt{1+\left(\psi_{i-12}+\psi_{i-13}\right)^{2}}+b_{i-1}+\right.\right.$ $\left.\left.\psi_{i-11}+\psi_{i-14}\right) / \lambda_{i-11}\right)$, such that

$$
\begin{aligned}
L V_{i-1} \leq & -\sum_{j=1}^{i-2}\left(c_{j}-\varepsilon_{j}-e_{j}\right) z_{j}^{4}-c_{i-1} z_{i-1}^{4}+d_{i-1} z_{i-1}^{3} z_{i} \\
& +\left(\hat{\theta}-\theta-\sum_{k=2}^{i-1} z_{k}^{3} \frac{\partial \alpha_{k}}{\partial \widehat{\theta}}\right)\left(\dot{\hat{\theta}}-\tau_{i-1}\right)
\end{aligned}
$$

where $V_{i-1}=\sum_{j=1}^{i-1}(1 / 4) z_{j}^{4}+(1 / 2)(\hat{\theta}-\theta)^{2}, \tau_{i-1}=\tau_{1}+$ $\sum_{k=2}^{i-1} z_{k}^{4}\left(\psi_{i-12}+\psi_{i-13}\right)$, and $\varepsilon_{j}=\sum_{k=1}^{j}\left(\varepsilon_{k 1}+\varepsilon_{k 2}+\varepsilon_{k 3}+\varepsilon_{k 4}\right)$, where $j=1, \ldots, n$.
Then, define the ith Lyapunov candidate function $V_{i}\left(\bar{z}_{i}, \widehat{\theta}\right)=V_{i-1}+(1 / 4) z_{i}^{4}$. From (16) and (22), it follows that

$$
\begin{aligned}
L V_{i} \leq & -\sum_{j=1}^{i-2}\left(c_{j}-\varepsilon_{j}-e_{j}\right) z_{j}^{4}-c_{i-1} z_{i-1}^{4}+d_{i-1} z_{i-1}^{3} z_{i} \\
& +z_{i}^{3}\left(d_{i} \eta_{i+1}+F_{i}\left(\bar{z}_{i}, x_{0}\right)-\frac{\partial \alpha_{i} \dot{\hat{\theta}}}{\partial \widehat{\theta}}\right. \\
& \left.-\frac{1}{2} \sum_{k, m=1}^{i-1} \frac{\partial^{2} \alpha_{i}}{\partial z_{k} \partial z_{m}} \phi_{k}^{T}\left(\bar{z}_{k}\right) \sum^{T}(t) \sum(t) \phi_{m}\left(\bar{z}_{m}\right)\right) \\
& +\frac{3}{2} z_{i}^{2} \operatorname{Tr}\left(G_{i}^{T}\left(\bar{z}_{i}\right) \sum^{T}(t) \sum(t) G_{i}\left(\bar{z}_{i}\right)\right) \\
& +\left(\hat{\theta}-\theta-\sum_{k=2}^{i-1} z_{k}^{3} \frac{\partial \alpha_{k}}{\partial \widehat{\theta}}\right)\left(\dot{\hat{\theta}}-\tau_{i-1}\right) .
\end{aligned}
$$

Using Lemmas 9 and 4, there are always known nonnegative smooth functions $\psi_{i 1}\left(\bar{z}_{i}\right), \psi_{i 2}\left(\bar{z}_{i}\right), \psi_{i 3}\left(\bar{z}_{i}\right), \psi_{i 4}\left(\bar{z}_{i}\right)$ and constant $\varepsilon_{i}>0, \varepsilon_{i j}>0$, where $i=1, \ldots, n$ and $j=1,2,3,4$.

Consider

$$
\begin{aligned}
z_{i}^{3} F_{i} & \leq\left|z_{i}^{3}\right|\left(\sum_{k=1}^{i-1}\left|z_{k}\right|\right) \gamma_{i 1}\left(\bar{z}_{i}, x_{0}\right) \\
& \leq \gamma_{i 1} z_{i}^{4}+\sum_{k=1}^{i-1}\left(\varepsilon_{k 1} z_{k}^{4}+\frac{3}{4}\left(4 \varepsilon_{k 1}\right)^{-1 / 3} \gamma_{i 1}^{4 / 3} z_{i}^{4}\right) \\
& \leq \sum_{k=1}^{i-1} \varepsilon_{k 1} z_{k}^{4}+\psi_{i 1} z_{i}^{4},
\end{aligned}
$$

where $\psi_{i 1} \geq \gamma_{i 1}+\sum_{k=1}^{i-1}(3 / 4)\left(4 \varepsilon_{k 1}\right)^{-1 / 3} \gamma_{i 1}^{4 / 3}$.

$$
\begin{aligned}
& -\frac{1}{2} z_{i}^{3} \sum_{k, m=1}^{i-1} \frac{\partial^{2} \alpha_{i}}{\partial z_{k} \partial z_{m}} \phi_{k}^{T} \sum^{T}(t) \sum(t) \phi_{m} \\
& \leq \frac{1}{2} z_{i}^{3} \sum_{k, m=1}^{i-1}\left|\frac{\partial^{2} \alpha_{i}}{\partial z_{k} \partial z_{m}}\right|\left(\sum_{j=1}^{k}\left|z_{j}\right|\right) \bar{p}_{k}\left(\bar{z}_{k}\right) \\
& \quad \times\left(\sum_{j=1}^{m}\left|z_{j}\right|\right) \bar{p}_{m}\left(\bar{z}_{m}\right)\left|\sum^{T}(t) \sum(t)\right| \\
& \leq z_{i}^{3}\left(\sum_{k=1}^{i-1} z_{k}^{2}\right) \overline{\bar{p}}_{i}\left(\bar{z}_{i}\right)\left|\sum^{T}(t) \sum(t)\right| \\
& \leq z_{i}^{4} \psi_{i 2}\left(\bar{z}_{i}\right) \theta+\sum_{k=1}^{i-1} \varepsilon_{k 2} z_{k}^{4},
\end{aligned}
$$

where $\psi_{i 2} \geq \sum_{k=1}^{i-1}(3 / 4)\left(4 \varepsilon_{k 2}\right)^{-1 / 3}\left(\overline{\bar{p}}_{i}\left(\bar{z}_{i}\right)\right)^{3 / 4}$.

$$
\begin{aligned}
& \frac{3}{2} z_{i}^{2} \operatorname{Tr}\left(G_{i}^{T} \sum^{T}(t) \sum(t) G_{i}\right) \\
& \quad \leq \frac{3}{2} z_{i}^{2} p_{i 2}^{2}\left(\bar{z}_{i}\right)\left(\sum_{k=1}^{i}\left|z_{k}\right|\right)^{2}\left|\sum^{T}(t) \sum(t)\right|
\end{aligned}
$$




$$
\begin{aligned}
& \leq \frac{3}{2} z_{i}^{2} i p_{i 2}^{2}\left(\bar{z}_{i}\right)\left(\sum_{k=1}^{i} z_{k}^{2}\right)\left|\sum^{T}(t) \sum(t)\right| \\
& \leq \frac{3}{2} z_{i}^{4} i p_{i 2}^{2}\left(\bar{z}_{i}\right) \theta+\sum_{k=1}^{i-1} \varepsilon_{k 3} z_{k}^{4}+\sum_{k=1}^{i-1} \frac{1}{4 \varepsilon_{k 3}}\left(\frac{3}{2} i p_{i 2}^{2}\right)^{2} z_{i}^{4} \theta \\
& \leq \sum_{k=1}^{i-1} \varepsilon_{k 3} z_{k}^{4}+\psi_{i 3} z_{i}^{4} \theta
\end{aligned}
$$

where $\psi_{i 3} \geq(3 / 2) i p_{i 2}^{2}+\sum_{k=1}^{i-1}\left(1 / 4 \varepsilon_{k 3}\right)\left((3 / 2) i p_{i 2}^{2}\right)^{2}$.

$$
\begin{aligned}
d_{i-1} z_{i-1}^{3} z_{i} & \leq \lambda_{i-12}\left|z_{i-1}^{3} z_{i}\right| \\
& \leq e_{i-1} z_{i-1}^{4}+\frac{1}{4}\left(\frac{4}{3} e_{i-1}\right)^{-3} z_{i}^{4} \lambda_{i 2}^{4} \\
& \leq e_{i-1} z_{i-1}^{4}+b_{i} z_{i}^{4}
\end{aligned}
$$

where $b_{i} \geq(1 / 4)\left((4 / 3) e_{i-1}\right)^{-3} \lambda_{i 2}^{4}, \tau_{i-1}=z_{1}^{4} p_{11}^{2}+\sum_{k=2}^{i-1} z_{k}^{4}\left(\psi_{k 2}+\right.$ $\left.\psi_{k 3}\right)$, and $\tau_{i}=\tau_{i-1}+\left(\psi_{i 2}+\psi_{i 3}\right) z_{i}^{4}$.

$$
\begin{aligned}
& -z_{i}^{3} \frac{\partial \alpha_{i}}{\partial \widehat{\theta}} \tau_{i} \\
& \leq z_{i}^{3}\left|\frac{\partial \alpha_{i}}{\partial \widehat{\theta}}\right|\left(\tau_{i-1}+z_{i}^{4}\left(\psi_{i 2}+\psi_{i 3}\right)\right) \\
& \leq z_{i}^{4} \sqrt{1+\left(z_{i}^{3} \frac{\partial \alpha_{i}}{\partial \widehat{\theta}}\right)^{2}}\left(\psi_{i 2}+\psi_{i 3}\right) \\
& +z_{i}^{3}\left|\frac{\partial \alpha_{i}}{\partial \widehat{\theta}}\right|\left(z_{1}^{4} p_{11}^{2}+\sum_{k=2}^{i-1} z_{k}^{4}\left(\psi_{k 2}+\psi_{k 3}\right)\right) \\
& +\frac{3}{4}(4)^{-1 / 3}\left(\left|\frac{\partial \alpha_{2}}{\partial \widehat{\theta}}\right| z_{1}^{3} p_{11}^{2}\right)^{4 / 3} \varepsilon_{i 4}{ }^{-1 / 3} z_{i}^{4} \\
& \leq \varepsilon_{i 4} z_{1}^{4}+\frac{3}{4}(4)^{-1 / 3} \sqrt{1+\left(\frac{\partial \alpha_{i}}{\partial \widehat{\theta}} z_{1}^{3} P_{11}^{2}\right)^{2}} \varepsilon_{i 4}^{-1 / 3} z_{i}^{4} \\
& +\sum_{k=2}^{i-1} \varepsilon_{k 4} z_{k}^{4} \\
& +\sum_{k=2}^{i-1} \frac{3}{4}(4)^{-1 / 3} \sqrt{1+\left(\frac{\partial \alpha_{i}}{\partial \widehat{\theta}} z_{k}^{3}\left(\psi_{k 2}+\psi_{k 3}\right)\right)^{2}} \varepsilon_{k 4}^{-1 / 3} z_{i}^{4} \\
& \leq \sum_{k=1}^{i-1} \varepsilon_{k 4} z_{k}^{4}+\psi_{i 4} z_{i}^{4}
\end{aligned}
$$

where $\psi_{i 4} \geq(3 / 4)\left(4 \varepsilon_{i 4}\right)^{-1 / 3} \sqrt{1+\left(\left(\partial \alpha_{i} / \partial \hat{\theta}\right) z_{1}^{3} P_{11}^{2}\right)^{2 / 3}}+$ $\sum_{k=2}^{i-1}(3 / 4)\left(4 \varepsilon_{k 4}\right)^{-1 / 3} \sqrt{1+\left(\left(\partial \alpha_{i} / \partial \hat{\theta}\right) z_{k}^{3}\left(\psi_{k 2}+\psi_{k 3}\right)\right)^{2}}$.

$$
\begin{gathered}
\alpha_{i+1}\left(\bar{z}_{i}, \widehat{\theta}\right)=-z_{i} \beta_{i}\left(\bar{z}_{i}, \widehat{\theta}\right) \\
\beta_{i}\left(\bar{z}_{i}, \widehat{\theta}\right)=\frac{c_{i}+\psi_{i 1}+\psi_{i 4}+b_{i}+\sqrt{1+\left(\psi_{i 2}+\psi_{i 3}\right)^{2}} \hat{\theta}}{\lambda_{i 1}}
\end{gathered}
$$

where $c_{i}>0$ is a design parameter to be chosen.

With the aid of (24)-(29) and (14), (23) can be simplified as

$$
\begin{aligned}
L V_{i} \leq & -\sum_{j=1}^{i-1}\left(c_{j}-\varepsilon_{j}-e_{j}\right) z_{j}^{4}-c_{i} z_{i}^{4} \\
& +d_{i} z_{i}^{3} z_{i+1}+\left(\hat{\theta}-\theta-\sum_{k=2}^{i} \frac{\partial \alpha_{k}}{\partial \widehat{\theta}} z_{k}^{3}\right)\left(\dot{\hat{\theta}}-\tau_{i}\right) .
\end{aligned}
$$

Finally, when $i=n, z_{n+1}=u$ is the actual control. By choosing the actual control law and the adaptive law,

$$
\begin{gathered}
u\left(\bar{z}_{n}, \hat{\theta}\right)=-z_{n} \beta_{n}\left(\bar{z}_{n}, \hat{\theta}\right), \\
\dot{\hat{\theta}}=\tau_{n}=z_{1}^{4} p_{11}^{2}+\sum_{k=2}^{n} z_{k}^{4}\left(\psi_{k 2}+\psi_{k 3}\right), \\
\beta_{n}\left(\bar{z}_{n}, \widehat{\theta}\right)=\frac{c_{n}+b_{n}+\psi_{n 1}+\psi_{n 4}+\sqrt{1+\left(\psi_{n 2}+\psi_{n 3}\right)^{2}} \hat{\theta}}{\lambda_{n 1}}, \\
u_{1}=e^{-\alpha t} u,
\end{gathered}
$$

where $c_{n}>0$ is a design parameter to be chosen and $\psi_{n i}, i=$ $1, \ldots 4$ are smooth functions; we get

$$
L V_{n} \leq-\sum_{j=1}^{n}\left(c_{j}-\varepsilon_{j}-e_{j}\right) z_{j}^{4},
$$

where $V_{n}(z, \widehat{\theta})=\sum_{k=1}^{n}(1 / 4) z_{k}^{4}+(1 / 2)(\widehat{\theta}-\theta)^{2}, z=$ $\left(z_{1}, \ldots z_{n}\right)$. We have finished the controller design procedure for $x_{0}\left(t_{0}\right) \neq 0$ and the parameter identification. Without loss of generality, we can assume that $t_{0} \neq 0$.

3.3. Switching Control and Main Result. In the preceding subsection, we have given controller design for $x_{0} \neq 0$. Now, we discuss how to choose the control laws $u_{0}$ and $u_{1}$ when $x_{0}=0$. We choose $u_{0}$ as $u_{0}=-\lambda_{0} x_{0}+u_{0}^{*}, u_{0}^{*}>0$. And choose the Lyapunov function $V_{0}=(1 / 2) x_{0}^{2}$. Its time derivative is given by $L V_{0}=-\lambda_{0} x_{0}^{2}+u_{0}^{*}$, which leads to the bounds of $x_{0}$. During the time period $\left[0, t_{s}\right)$, using $u_{0}=-\lambda_{0} x_{0}+u_{0}^{*}$, new control law $u$ can be obtained by the control procedure described above to the original $x$-subsystem in (1). Then, we can conclude that the $x$-state of (1) cannot be blown up during the time period $\left[0, t_{s}\right)$. Since at $x\left(t_{s}\right) \neq 0$, we can switch the control inputs $u_{0}$ and $u$ to (6) and (31), respectively.

Now, we state the main results as follows.

Theorem 11. Under Assumption 5, if the proposed adaptive controller (31) together with the above switching control strategy is used in (1), then for any initial contidion $\left(x_{0}, x, \widehat{\theta}\right) \in R^{n}$, 
the closed-loop system has an almost surely unique solution on $[0, \infty)$, the solution process is bounded in probability, and $P\left\{\lim _{t \rightarrow \infty} \hat{\theta}(t)\right.$ exists and is finite $\}=1$.

Proof. According to the above analysis, it suffices to prove in the case $x_{0}(0) \neq 0$. Since we have already proven that $x_{0}$ can be globally exponentially convergent to zero in probability in Section 3.1, we only need prove that $x(t)$ is convergent to zero in probability also. In this case, we choose the Lyapunov function $V=V_{n}$, and $c_{i}>\varepsilon_{i}+e_{i}$; from (32) and Lemma 3, we know that the closed-loop system has an almost surely unique solution on $[0, \infty)$, and the solution process is bounded in probability.

\section{Conclusions}

This paper investigates the globally exponential stabilization problem for a class of stochastic nonholonomic systems in chained form. To deal with the nonlinear parametrization problem, a parameter separation technique is introduced. With the help of backstepping technique, a smooth adaptive controller is constructed which ensures that the closed-loop system is globally asymptotically stable in probability. A further work is how to design the output-feedback tracking control for more high-order stochastic nonholonomic systems.

\section{Acknowledgments}

This work was supported by the university research projects of Department of Education in Shandong Province, China (J13LI03). The author would like to thank the reviewers for their helpful comments.

\section{References}

[1] R. W. Brockett, R. S. Millman, and H. J. Sussmann, Differential Geometric Control Theory, Birkhauser, Basel, Switzerland, 1983.

[2] Z. Sun, S. S. Ge, W. Huo, and T. H. Lee, "Stabilization of nonholonomic chained systems via nonregular feedback linearization," Systems and Control Letters, vol. 44, no. 4, pp. 279289, 2001.

[3] Z. P. Jiang, "Robust exponential regulation of nonholonomic systems with uncertainties," Automatica, vol. 36, no. 2, pp. 189209, 2000.

[4] Z. Xi, G. Feng, Z. P. Jiang, and D. Cheng, "Output feedback exponential stabilization of uncertain chained systems," Journal of the Franklin Institute, vol. 344, no. 1, pp. 36-57, 2007.

[5] X. Y. Zheng and Y. Q. Wu, "Adaptive output feedback stabilization for nonholonomic systems with strong nonlinear drifts," Nonlinear Analysis: Theory, Methods and Applications, vol. 70, no. 2, pp. 904-920, 2009.

[6] F. Z. Gao, F. S. Yuan, and H. J. Yao, "Robust adaptive control for nonholonomic systems with nonlinear parameterization," Nonlinear Analysis: Real World Applications, vol. 11, no. 4, pp. 3242-3250, 2010.

[7] M. Krstic and H. Deng, Stabilization of Nonlinear Uncertain Systems, Springer, New York, NY, USA, 1998.

[8] H. Deng, M. Krstic, and R. J. Williams, "Stabilization of stochastic nonlinear systems driven by noise of unknown covariance,"
Proceedings of the IEEE Transactions on Automatic Control, vol. 46, no. 8, pp. 1237-1253, 2001.

[9] X. Yu and X. J. Xie, "Output feedback regulation of stochastic nonlinear systems with stochastic iISS inverse dynamics," Proceedings of the IEEE Transactions on Automatic Control, vol. 55, no. 2, pp. 304-320, 2010.

[10] X. J. Xie, N. Duan, and X. Yu, "State-feedback control of high-order stochastic nonlinear systems with SiISS inverse dynamics," Proceedings of the EEE Transactions on Automatic Control, vol. 56, no. 8, pp. 1921-1926, 2011.

[11] N. Duan and X. J. Xie, "Further results on output-feedback stabilization for a class of stochastic nonlinear systems," Proceedings of the IEEE Transactions on Automatic Control, vol. 56, no. 5, pp. 1208-1213, 2011.

[12] X. Yu, X. J. Xie, and Y. Q. Wu, "Decentralized adaptive output-feedback control for stochastic interconnected systems with stochastic unmodeled dynamic interactions," International Journal of Adaptive Control and Signal Processing, vol. 25, no. 8, pp. 740-757, 2011.

[13] L. Liu and X. J. Xie, "State-feedback stabilization for stochastic high-order nonlinear systems with SISS inverse dynamics," Asian Journal of Control, vol. 14, no. 4, pp. 1-11, 2012.

[14] X. J. Xie and L. Liu, "A homogeneous domination approach to state feedback of stochastic high-order nonlinear systems with time-varying delay," Proceedings of the IEEE Transactions on Automatic Control, vol. 58, no. 2, pp. 494-499, 2013.

[15] Z. J. Wu, Y. Q. Xia, and X. J. Xie, "Stochastic barbalat's lemma and its applications," Proceedings of the IEEE Transactions on Automatic Control, vol. 57, no. 6, pp. 1537-1543, 2012.

[16] X. Y. Qin, "State-feedback stablization for a class of highorder stochastic nonlinearsystems," Journal of Anhui University (Natural Science Edition), vol. 36, no. 4, pp. 7-12, 2012.

[17] J. Wang, H. Gao, and H. Li, "Adaptive robust control of nonholonomic systems with stochastic disturbances," Science in China F, vol. 49, no. 2, pp. 189-207, 2006.

[18] F. Z. Gao, F. S. Yuan, and H. J. Yao, "Adaptive Stabilization for a class of Stochastic Nonholonomic Systems with nonlinear parameterization," in Proceedings of the 24th Chinese Control and Decision Conference (CCDC '12), pp. 1377-1382, Taiyuan, China, 2012.

[19] Y. L. Liu and Y. Q. Wu, "Output feedback control for stochastic nonholonomic systems with growth rate restriction," Asian Journal of Control, vol. 13, no. 1, pp. 177-185, 2011.

[20] W. Lin and C. Qian, "Adaptive control of nonlinearly parameterized systems: a nonsmooth feedback framework," Proceedings of the IEEE Transactions on Automatic Control, vol. 47, no. 5, pp. 757-774, 2002. 


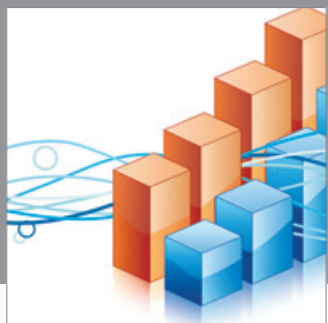

Advances in

Operations Research

mansans

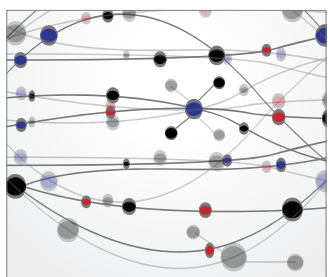

The Scientific World Journal
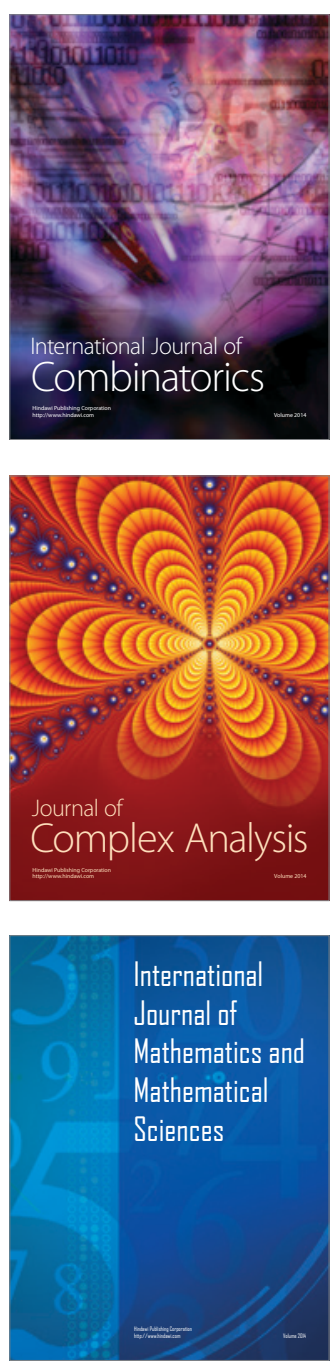
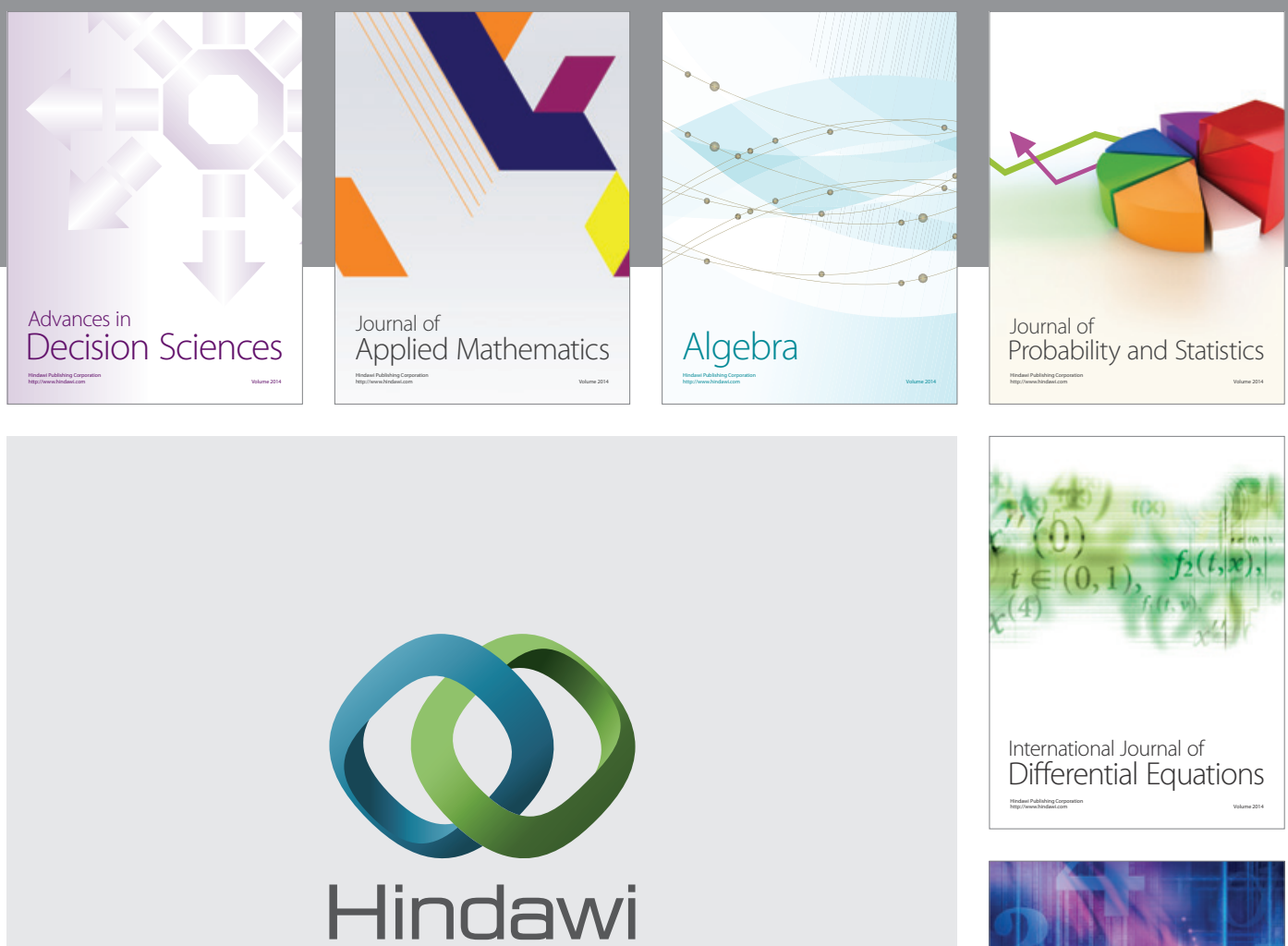

Submit your manuscripts at http://www.hindawi.com
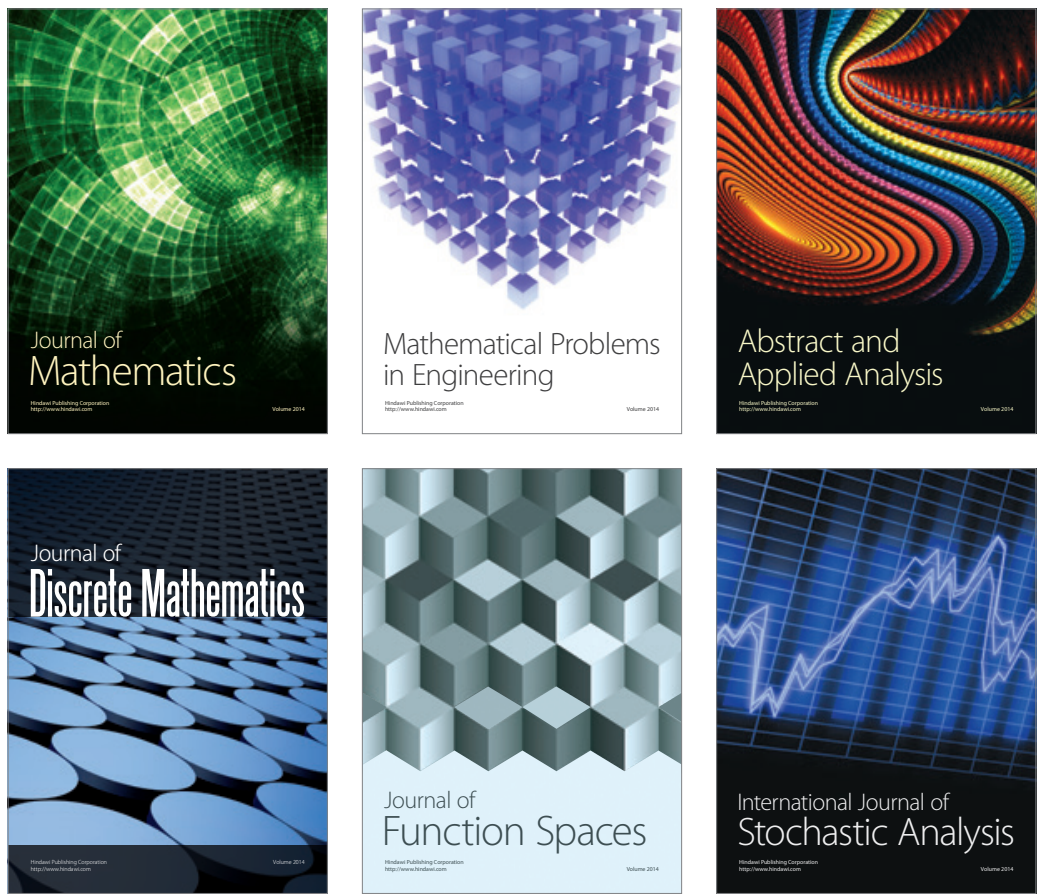

Journal of

Function Spaces

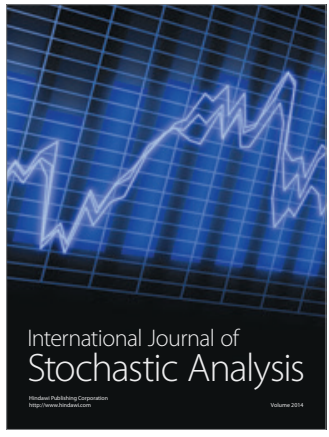

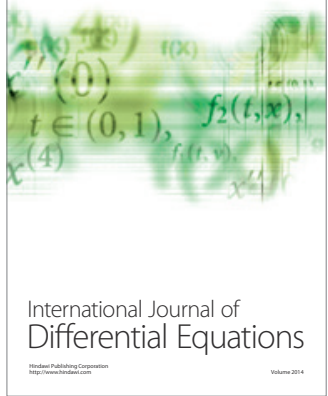
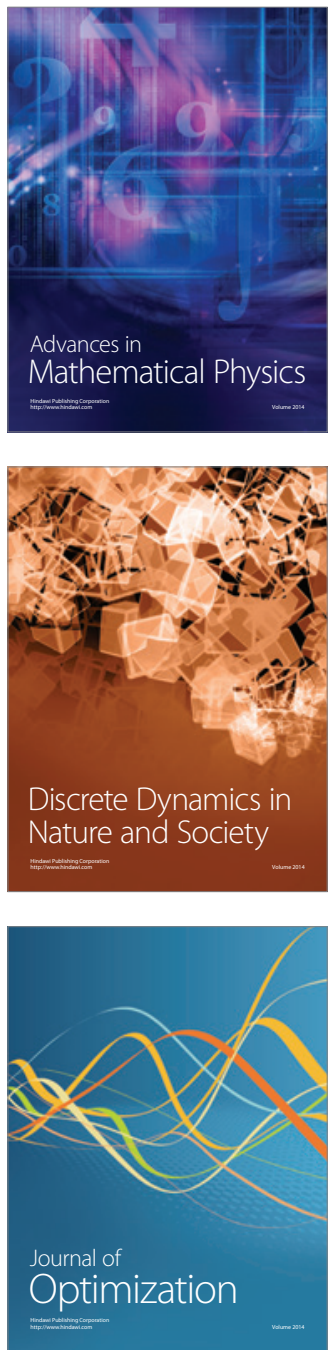